\title{
Therapeutic Effects of Transplantation of As-MiR-937-Expressing Mesenchymal Stem Cells in Murine Model of Alzheimer's Disease
}

\author{
Zhen Liu Cunfu Wang Xiao Wang Shunliang Xu \\ Department of Neural Medicine, Second Hospital of Shandong University, Jinan, China
}

\section{Key Words}

Alzheimer's disease (AD) • Mesenchymal stem cells (MSCs) • Amyloid-beta peptide aggregates $(A \beta) \cdot$ Brain-derived neurotrophic factor (BDNF) • Brn-4 $\bullet$ MiR-937

\begin{abstract}
Background/Aims: Alzheimer's disease (AD) is one of the most common dementias among aged people, and is clinically characterized by progressive memory loss, behavioral and learning dysfunction and cognitive deficits. So far, this is no cure for AD. A therapeutic effect of transplantation of mesenchymal stem cells (MSCs) into murine model of $A D$ has been reported, but remains to be further improved. Brn-4 is a transcription factor that plays a critical role in neuronal development, whereas the effects of Brn-4 overexpression in transplanted MSCs on AD are unknown. Methods: MSCs were isolated from mouse bone marrow and induced to overexpress antisense of miRNA-937 (as-miR-937) through adeno-associated virus (AAV)-mediated transduction, and purified by flow cytometry based on expression of a GFP co-transgene in the cells. The Brn-4 levels in mouse MSCs were examined in miR-937modified MSCs by RT-qPCR and by Western blot. These miR-937-modified MSCs were then transplanted into an APP/PS1 transgenic AD model in mice. The effects of saline control, MSCs and asmiR-937 MSCs on AD mice were examined by deposition of amyloid-beta peptide aggregates $(A \beta)$, social recognition test (SR), Plus-Maze Discriminative Avoidance Task (PMDAT) and the levels of Brain-derived neurotrophic factor (BDNF) in the mouse brain. Results: MSCs expressed high levels of Brn-4 transcripts but low levels of Brn-4 protein. Poor protein vs mRNA levels of Brn-4 in MSCs appeared to result from the presence of high levels of miR-937 in MSCs. miR-937 inhibited translation of Brn-4 mRNA through binding to the 3'-UTR of the Brn-4 mRNA in MSCs. Expression of as-miR-937 significantly increased Brn-4 protein levels in MSCs. Transplantation of as-miR-937-expressing MSCs significantly reduced the deposition of $A B$, increased the levels of BDNF, and significantly improved the appearance in SR and PM-DAT in AD mice. Conclusion: Overexpression of as-miR-937 in MSCs may substantially improve the therapeutic effects of MSCs on $A D$, possibly through augmenting Brn-4 levels in MSCs.

Copyright $\odot 2015$ S. Karger AG, Basel

Shunliang Xu

Department of Neural Medicine, Second Hospital of Shandong University, 247 Beiyuan Road, Jinan 250033, (China)

Tel. +8653185875469, E-Mail shunliang_xu@163.com
\end{abstract}

KARGER 125 


\section{Cellular Physiology Cell Physiol Biochem 2015;37:321-330 \begin{tabular}{l|l} 
and Biochemistry Published onlIne: August 24, 2015 & $\begin{array}{l}\text { C) 2015 S. Karger AG, Basel } \\
\text { www.karger.com/cpb }\end{array}$ \\
\hline
\end{tabular} \\ Liu/Wang/Wang/Xu: Therapy of AD by As-MiR-937-Expressing MSCs}

\section{Introduction}

Alzheimer's disease (AD) is one of the most common dementias among aged people and it is clinically characterized by progressive memory loss, behavioral and learning dysfunction and cognitive deficits [1-3]. The pathological characterization of AD includes extracellular senile plaques [composed of amyloid-beta peptide aggregates $(A \beta)$ ], intracellular neurofibrillary tangles, synaptic dysfunction, and the loss of neurons in the brain [1-3]. The neuronal cell death leads to a reduction in size of the temporal and frontal lobes of the brain, which are responsible for learning, memorization and other mental functions [1-3]. These pathological changes finally result in progressive memory and learning dysfunction and cognitive impairment in AD patients [1-3]. The APP/PS1 transgenic mouse is a murine AD model with similar characteristics, including social memory impairment, senile plaque formation and vascular deficits in AD patients [4-7].

It was previously believed that the adult mammalian brain was devoid of stem cells that could regenerate after injury. However, recent studies have shown that neurogenesis may occur throughout the lifespan of adult mammals, as an alternative way to replace the lost neurons by replication of remaining ones [8-12]. Nevertheless, there is no cure for AD right now.

Therapeutic effects of transplantation of mesenchymal stem cells (MSCs) into a murine model of AD have been reported [13-17]. These studies suggest that the transplantation of MSCs can stimulate neurogenesis in the brains of adult rodents. Transplantation of MSCs could facilitate functional recovery in animal models of neurological disorders by promoting neurogenesis, via either enhanced proliferation of endogenous neural stem cells in the subgranular zone of the dentate gyrus [14], or via increases in the proliferation and neural differentiation of newly generated cells in the subventricular zone [15]. However, the outcome of the therapeutic effects of MSCs on neurogenesis and recovery of brain function is not sufficient, and remains to be further improved. Brn-4 is a transcription factor that plays a critical role in neuronal development [18-21], whereas the effects of Brn-4 overexpression in transplanted MSCs on AD are unknown. Therefore, increasing the levels of Brn-4 in MSCs may be examined to evaluate its effects on the outcome of MSC transplantation.

Here, we found that MSCs expressed high levels of Brn-4 transcripts but low levels of Brn-4 protein. Poor protein vs mRNA levels of Brn-4 in MSCs appeared to result from the presence of high levels of miRNA (miR)-937 in MSCs. miR-937 inhibited translation of Brn-4 mRNA through binding to the 3'-UTR of the Brn-4 mRNA in MSCs. Expression of asmiR-937 significantly increased Brn-4 protein levels in MSCs. Transplantation of as-miR937-expressing MSCs significantly reduced the deposition of $A \beta$, increased the levels of BDNF, and significantly improved the appearance in SR and PM-DAT in AD mice. Together, these data suggest that overexpression of as-miR-937 in MSCs may substantially improve the therapeutic effects of MSCs on AD, possibly through augmenting Brn-4 levels in MSCs. Our study should have therapeutic implications for treating AD.

\section{Materials and Methods}

\section{Animals}

C57BL/6 and transgenic APP/PS1 mice expressing the human APPswe (K595N/M596L) and presenilin 1 (PS1 $\triangle \mathrm{E} 9$ ) mutants were purchased from Jackson Laboratories (Bar Harbor, ME, USA). These transgenic mice were maintained on their original genetic background until the age of 8 months, when they developed $\mathrm{A} \beta$ deposits and exhibited significant cognitive impairment. The mice were raised at $22 \pm 1^{\circ} \mathrm{C}$, under a 12 hour light/dark cycle, with free access to standard food and water. All experimental procedures were in strict accordance with the Guidance Suggestions for the Care and Use of Laboratory Animals, issued by the Ministry of Science and Technology of China, and proved by the research committee of Second Hospital of Shandong University. 


\section{Cellular Physiology Cell Physiol Biochem 2015;37:321-330 \begin{tabular}{ll|l} 
and Biochemistry Publisned onIIne: August 24, 2015 & $\begin{array}{l}\text { C 2015 S. Karger AG, Basel } \\
\text { www.karger.com/cpb }\end{array}$ \\
\hline
\end{tabular}}

Liu/Wang/Wang/Xu: Therapy of AD by As-MiR-937-Expressing MSCs

Preparation, differentiation and Transduction of MSCs

Bone-marrow derived MSCs from 8-week-old C57BL/6 mice (Jackson Laboratories) were collected from femurs and tibias by flushing with culture medium (DMEM, Dulbecco's Modified Eagle's Medium, Gibco, San Diego, CA, USA). The cells were centrifuged and resuspended in DMEM low glucose containing inactivated 10\% fetal bovine serum (FBS), Gibco, 3.7g/l HEPES (N-2-hydroxyethylpiperazine-N'-2-ethanesulphonic acid, Sigma-Aldrich, St. Louis, MO, USA), 1\% 200 mmol/l L-glutamine 100x (Gibco) and 1\% PSA (Gibco). The cell number and viability were determined by trypan blue staining (Gibco) and reached a final cell density of $5 \times 10^{6}$ cells $/ \mathrm{ml}$. The cells were incubated in a humidified chamber with $5 \% \mathrm{CO}_{2}$ at $37^{\circ} \mathrm{C}$ for $72 \mathrm{~h}$, and the adherent cells, which were considered MSCs, were maintained in culture until reaching $\sim 80 \%$ semi-confluence. Then, the MSCs were washed, incubated with trypsin-ethylenediaminetetraacetic acid (EDTA) (StemCell Technologies, Vancouver, Canada) and prepared to be frozen with a cryoprotectant solution of dimethylsulphoxide (DMSO, MP Biomedicals, Santa Ana, USA) and FBS.

MSCs were transduced with an adeno-associated virus (AAV) carrying as-miR-937 and GFP construct (connected with a 2A sequence), or control AAV (null and GFP sequences; null). The sequence for asmiR-937 is 5'-GGCAGAGAGUCAGAGCGCGGAU-3'. GFP allows purification of the transduced cells by flow cytometry based on green fluorescence. Human embryonic kidney 293 cell line (HEK293) was used for virus production. In this study we used a pAAV-CMV-GFP plasmid (Clontech, Mountain View, CA, USA), a packaging plasmid carrying the serotype 8 rep and cap genes, and a helper plasmid carrying the adenovirus helper functions (Applied Viromics, LLC. Fremont, CA, USA). AAVs was prepared by triple transfection of the newly prepared plasmids, R2C8 (containing AAV2 Rep and AAV8 capsid genes) and plAd5 (containing adenovirus helper genes) into HEK293 cells by Lipofectamine 2000 reagent (Invitrogen, St. Louis, MO, USA). The viruses were purified using $\mathrm{CsCl}$ density centrifugation and then titered by a quantitative densitometric dot-blot assay. Then, the MSCs cells were incubated with AAVs at a MOI of 100 for 12 hours to transduce the cells. Afterwards, transduced cells were purified by flow cytometry base on GFP expression.

A positive clone was selected after subjection to chondrogenetic, osteogenic, and adipogenic differentiation assays to confirm phenotype. For chondrogenetic induction, $2.5 \times 10^{5}$ MSCs were induced with $5 \mathrm{ml}$ chondrogenetic induction medium containing $10 \mu \mathrm{g}$ transforming growth factor $\beta 1$ (R\&D

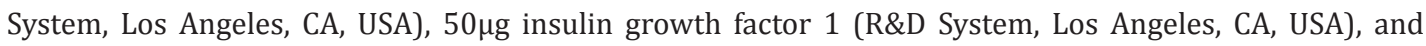
$2 \mathrm{mg} / \mathrm{ml}$ dexamethasone (DMSO, Sigma-Aldrich, St. Louis, MO, USA) followed by centrifugation at $500 \mathrm{~g}$ for $5 \mathrm{~min}$. The cell pellets were maintained in the chondrogenetic induction medium for 14 days and subjected to Alcian blue staining. For osteogenic induction, cells were digested and seeded onto a 24well plate at a density of $10^{4}$ cells/well, and then maintained in osteogenic induction medium containing 10nM Vitamin D3 (Sigma-Aldrich) and $10 \mathrm{mM} \beta$-phosphoglycerol and $0.1 \mu \mathrm{M}$ DMSO for 14 days and were subjected to Von kossa staining. For adipogenic induction, cells were digested and seeded onto a 24-well plate at a density of $10^{4}$ cells / well and then maintained in the adipogenic induction medium containing 0.5mmol/l 3-isobutyl-1-methylxanthine (IBMX), 200 $\mathrm{mol} / \mathrm{l}$ indomethacin, $10 \mu \mathrm{mol} / \mathrm{l}$ insulin and 1 mol/l DMSO for 14 days and subjected to Oil red 0 staining.

\section{Cell Transplantation}

The APP/PS1 mice at 9 months of age ( $\mathrm{n}=10$ per group) were anesthetized, and $10^{6}$ of the as-miR937-MSCs or control MSCs in a $5 \mu$ l PBS were injected into the bilateral hippocampi with an automated infusion pump $(1 \mu \mathrm{l} / \mathrm{min}$; PHD 2000, Harvard Apparatus, Holliston, MA, USA). The stereotaxic coordinates were as follows: $2 \mathrm{~mm}$ posterior to the bregma, $2 \mathrm{~mm}$ bilateral from the midline, and $2 \mathrm{~mm}$ ventral to the skull surface. In another control group, PBS vehicle alone was injected into the hippocampi of APP/PS1 mice at the same time. After surgery, the mice were kept warm until recovery from anesthesia, and then returned to their home cages.

\section{Western blot}

The protein was extracted from the mouse brain tissue and homogenized in RIPA lysis buffer $(1 \%$ NP40, $0.1 \%$ SDS, $100 \mu \mathrm{g} / \mathrm{ml}$ phenylmethylsulfonyl fluoride, $0.5 \%$ sodium deoxycholate, in PBS) on ice. The supernatants were collected after centrifugation at $12000 \times \mathrm{g}$ at $4^{\circ} \mathrm{C}$ for $20 \mathrm{~min}$. Protein concentration was determined using a BCA protein assay kit (Bio-rad, China), and whole lysates were mixed with $4 \times$ SDS loading buffer (125mmol/l Tris-HCl, 4\% SDS, $20 \%$ glycerol, $100 \mathrm{mmol} / \mathrm{l}$ DTT, and $0.2 \%$ bromophenol blue) at a ratio of $1: 3$. Protein samples were heated at $100^{\circ} \mathrm{C}$ for $5 \mathrm{~min}$ and were separated on SDS-polyacrylamide 


\section{Cellular Physiology Cell Physiol Biochem 2015;37:321-330 \begin{tabular}{ll|l} 
and Biochemistry & $\begin{array}{l}\text { DOI 10.1159/000430356 } \\
\text { Publisned onine: August 24, } 2015\end{array}$ & $\begin{array}{l}\text { O) 2015 S. Karger AG, Basel } \\
\text { www.karger.com/cpb }\end{array}$ \\
\cline { 2 - 3 }
\end{tabular}}

Liu/Wang/Wang/Xu: Therapy of AD by As-MiR-937-Expressing MSCs

gels. The separated proteins were then transferred to a PVDF membrane. The membrane blots were first probed with a primary antibody. After incubation with horseradish peroxidase-conjugated second antibody, autoradiograms were prepared using the enhanced chemiluminescent system to visualize the protein antigen. The signals were recorded using X-ray film. Primary antibodies for Western Blot are rat anti-Brn-4, anti-brain-derived neurotrophic factor (BDNF) and rabbit anti- $\beta$-actin (Cell Signaling, San Jose, CA, USA). $\beta$-actin was used as a protein loading control. The secondary antibody was HRP-conjugated anti-rabbit (Jackson ImmunoResearch Labs, West Grove, PA, USA). Images shown in the figures were representative of 5 individuals. NIH ImageJ software (Bethesda, MA, USA) was used for image acquisition and densitometric analysis of the gels.

\section{Quantitative PCR (RT-qPCR)}

MiRNA and total RNA were extracted from mouse brain with miRNeasy mini kit or RNeasy kit (Qiagen, Hilden, Germany), respectively. Quantitative PCR was performed in duplicates with QuantiTect SYBR Green PCR Kit (Qiagen). All primers were purchased from Qiagen. Values of genes were normalized against $\beta$-actin, and then compared to controls.

ELISA

The concentration of VEGF-A was determined by human VEGF-A ELISA Kit (R\&D System, Los Angeles, CA, USA). ELISAs were performed according to the instructions of the manufacturer. Briefly, the collected condition media was added to a well coated with primary antibody, and then immunosorbented by biotinylated primary antibody at room temperature for 2 hours. The color development catalyzed by horseradish peroxidase was terminated with $2.5 \mathrm{~mol} / \mathrm{l}$ sulfuric acid and the absorption was measured at $450 \mathrm{~nm}$. The protein concentration was determined by comparing the relative absorbance of the samples with the standards.

\section{Luciferase-reporter activity assay}

Luciferase-reporters were successfully constructed using molecular cloning technology. Target sequence was inserted into pGL3-Basic vector (Promega, Madison, WI, USA) to obtain pGL3-Brn-4-3'UTR, which contains the miR-937 binding sequence (Brn-4-3'UTR sequence). A172-miR-937, A172-null, or A172-as-miR-937 cells were seeded in 24-well plates for 24 hours, after which they were transfected with $1 \mu \mathrm{g}$ of Luciferase-reporter plasmids per well using PEI Transfection Reagent. Then luciferase activities were measured using the dual-luciferase reporter gene assay kit (Promega), according to the manufacturer's instructions.

\section{Social Recognition Test (SR)}

The mice were tested in the social recognition test (SR) to assess their social recognition memory and novelty reaction. Seven days before the SR test, the animals were kept in individual cages to establish territorial dominance. Six-week-old Swiss male mice were used as intruders. Before the first trial, an empty chamber was placed in the test cage with the subject mouse to allow spontaneous exploration (Fig. 1). During an "initial encounter", an intruder was placed inside a transparent acrylic chamber with several orifices on the walls. The sessions consisted of five trials of $5 \mathrm{~min}$ each, separated by $10 \mathrm{~min}$ intervals. In the subsequent four trials, the subject mouse was exposed to the same intruder. In the last trial (5th), a new intruder (2nd intruder) was placed in the same acrylic chamber (which was properly cleaned to remove the odor of the previous intruder), and the time spent sniffing was quantified again. The time spent sniffing in the social interactions was scored with a stopwatch by an observer blinded to the phenotype or treatment. The duration of investigation by the host mouse, consisting of sniffing the intruder through the orifices, was summed over the course of the trial and was used as a measure of social recognition. A reduction in the time spent sniffing between the 1 st and 4 th trials indicated social recognition. An increase in the time spent sniffing in the 5 th trial compared to the 4 th trial indicated reaction to the novelty.

Plus-Maze Discriminative Avoidance Task (PM-DAT)

The apparatus employed in the plus-maze discriminative avoidance task (PM-DAT) is a modified elevated plus-maze made of wood. The apparatus has two enclosed arms with sidewalls and no top (28.5 $\times 7 \times 18.5 \mathrm{~cm})$. The enclosed arms are opposite to two open arms $(28.5 \times 7 \mathrm{~cm})$. A non-illuminated, $100 \mathrm{~W}$ 
lamp and a hair dryer were placed over the center of one of the enclosed arms (aversive enclosed arm). In the training session, each mouse was placed at the center of the apparatus, and during a 10 min period, the aversive stimuli were administered every time the animal entered the enclosed arm containing the lamp and the hair dryer and was continued until the animal left the arm. The aversive stimuli consisted of both the illumination of the $100 \mathrm{~W}$ light and cold air blow produced by the hair dryer. In the test session, which was performed in the same room $24 \mathrm{~h}$ after the training, mice were again placed in the center of the apparatus and were observed for $3 \mathrm{~min}$; however, the mice did not receive the aversive stimuli when they entered the aversive enclosed arm even though the non-illuminated lamp and the hair dryer were still placed on the middle of this arm to help distinguish between the aversive and non-aversive arms. In all experiments, the animals were observed in a blind manner, and the apparatus was cleaned with a $5 \%$ alcohol solution after each behavioral session. The percentage of time spent in the aversive enclosed arm (time spent in aversive enclosed arm/time spent in both enclosed arms $\times 100$ ) was calculated. Learning and memory were evaluated by the percentage of time spent in the aversive enclosed arm during training and testing, respectively. All the measures taken during the PM-DAT were obtained manually.

\section{Statistics}

All statistical analyses were carried out using the SPSS 19.0 statistical software package. All values are depicted as mean \pm standard deviation from 5 individuals and are considered significant if $p<0.05$. All data were statistically analyzed using one-way ANOVA with a Bonferoni correction, followed by Fisher's Exact Test upon necessity.

\section{Results}

\section{Preparation of miR-937-depleted MSCs}

The therapeutic effects of MSCs on AD require further improvement. Since Brn-4 is a critical transcription factor for neuronal development, and since we found that MSCs express high levels of Brn-4 mRNA and very low levels of Brn-4 protein, we hypothesized that Brn4 translation may be regulated, e.g. by microRNAs, in MSCs. Among all Brn-4-targeting miRNAs, we specifically found that miR-937, which bound to 3'UTR of Brn-4 mRNA at 40th46th base site (Fig. 1A), was highly expressed in MSCs. Thus, we transduced isolated primary mouse MSCs with antisense of miR-937 (as-miR-937) by AAV. The virus also carries a GFP reporter, and all the transduced cells expressed GFP (Fig. 1B). The control MSCs received AAV-null. These GFP-expressing transduced cells were then purified by flow cytometry (Fig.

Fig. 1. Preparation of miR-937-depleted MSCs. (A) Bioinformatics showing that miR-937 binds to 3'UTR of Brn-4 mRNA at 40th-46th base site in MSCs. (B) transduced primary mouse MSCs with antisense of miR-937 (as-miR-937) by AAV in culture. The virus also carries a GFP reporter, and all the transduced cells expressed GFP. The control MSCs received AAV-null. (C) The GFP-expressing transduced cells were purified by flow cytometry, shown by representative flow chart. Scale bar is $20 \mu \mathrm{m}$.
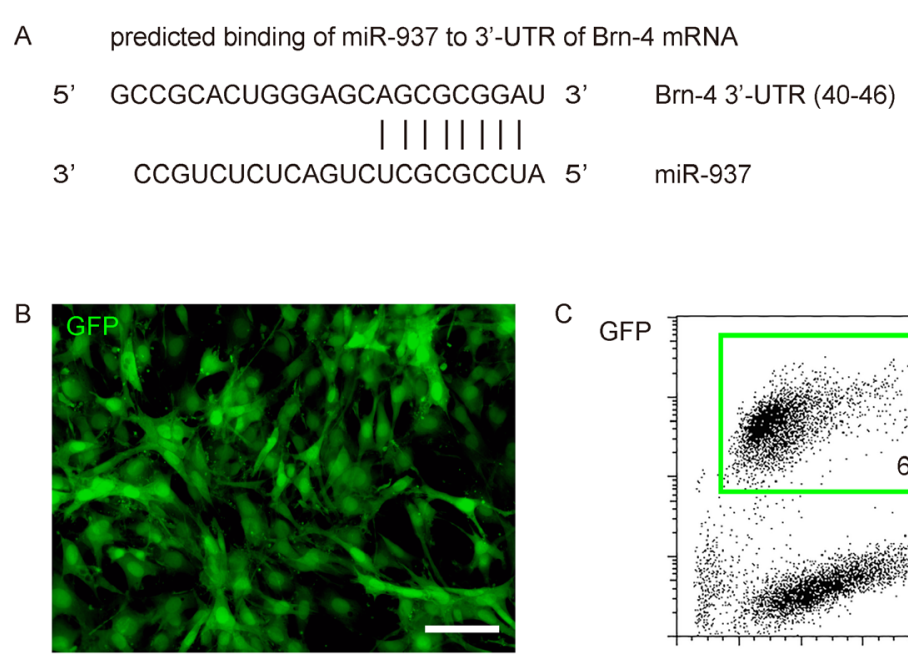

as-miR-937-MSCs

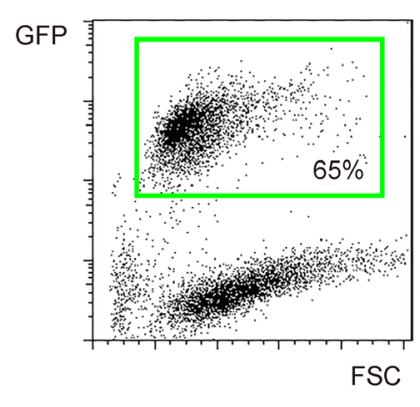


Fig. 2. As-miR-937-MSCs expresses increased levels of Brn-4. (A) The levels of miR-937 levels in transduced MSCs. (B-C) The Brn-4 levels in transduced MSCs, by mRNA (B), and by Western blot (C). (D-F) Differentiation assays to confirm the MSC-phenotype after miR-937 modulation. (D) Von kossa staining to evaluate osteogenic induction. (E) Oil red 0 staining to evaluate adipogenic induction. (F) Alcian blue staining to evaluate chondrogenetic induction. ${ }^{*} \mathrm{p}<0.05$. NS: non-significant. $\mathrm{N}=10$. Scale bars are $50 \mu \mathrm{m}$.

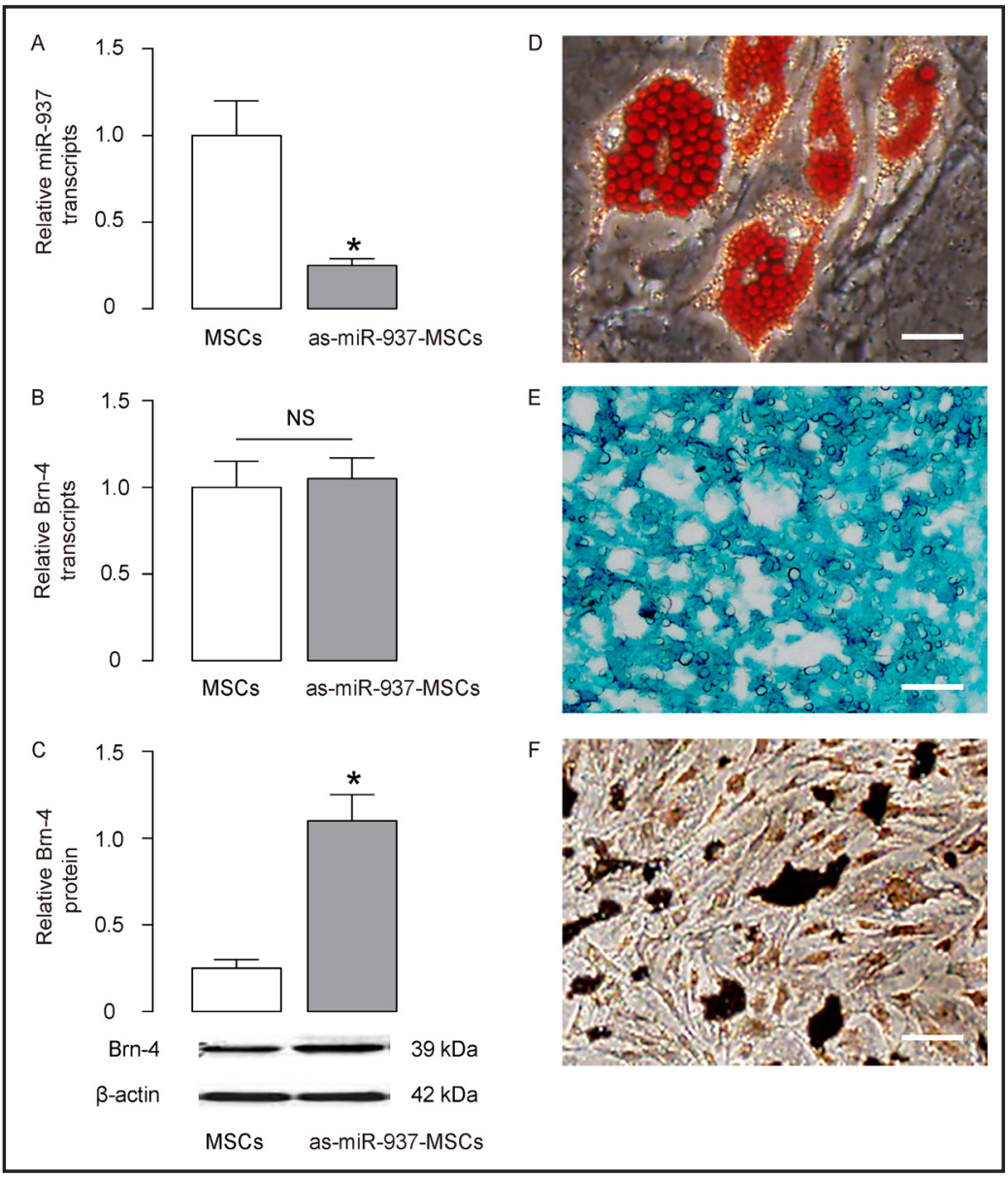

1C). The growth rate of as-miR-937-MSCs was compared with MSCs, and was found not to be different.

As-miR-937-MSCs express increased levels of Brn-4

First, we examined the miR-937 levels in these transduced cells and confirmed the knockdown of miR-937 in as-miR-937-MSCs, compared to control AAV-null-transduced MSCs (MSCs, Fig. 2A). Then we analyzed Brn-4 levels in these MSCs, and found that although the mRNA levels of Brn-4 in as-miR-937-MSCs cells were unchanged (Fig. 2B), the protein of Brn-4 in as-miR-937-MSCs cells were significantly increased (by more than 3 folds, Fig. 2C). These data suggest that depletion of miR-937 in MSCs significantly increases Brn-4 protein expression in MSCs. Then these transduced MSCs were subjected to differentiation assays to confirm the MSC-phenotype after miR-937 modulation. We performed Von kossa staining to evaluate osteogenic induction, Oil red 0 staining to evaluate adipogenic induction and Alcian blue staining to evaluate chondrogenetic induction (Fig. 2D-F). Our data confirmed the maintenance of the MSC phenotype of these transduced MSCs (as-miR-937-MSCs and MSCs).

Transplantation of as-miR-937-MSCs further improves the recovery of Social Recognition Memory in AD mice

Then we evaluated the effects of transplantation of as-miR-937-MSCs, or MSCs on the recovery of Social Recognition Memory in AD mice, compared to AD mice that received saline as a control (saline), at 9 months of age. Measurements for sniffing durations with treatment as a between-subject and time as a within-subject showed that in all experimental groups, the mice recognized the same intruder and showed elevated sniffing times when the intruder mouse was changed in the 5th trial, which was indicative of intense exploratory 
Fig. 3. Transplantation of as-miR937-MSCs further improves the recovery of Social Recognition Memory in AD mice. We evaluated the effects of transplantation of as-miR-937-MSCs, or MSCs on the recovery of Social Recognition Memory in AD mice, compared to $\mathrm{AD}$ mice that received saline as a control (saline), at 9 months of age. Measurements for sniffing durations with treatment as a between-subject and time as a wi-

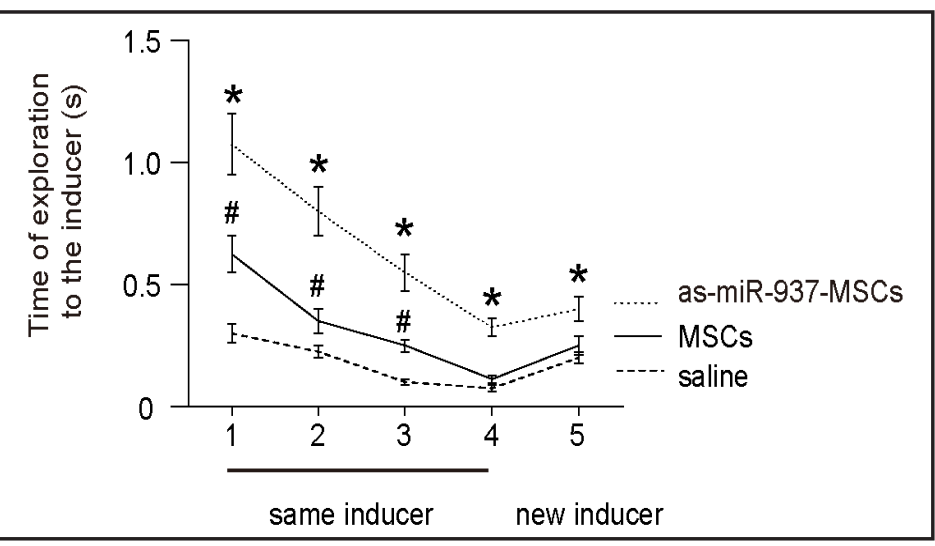
thin-subject showed that in all experimental groups, the mice recognized the same intruder and showed elevated sniffing times when the intruder mouse was changed in the 5th trial, which was an indicative of intense exploratory behavior and innate interest. The mice that received as-miR-937-MSCs mice showed further improvement in the test, compared to the mice that received MSCs, which had already better performance, compared to the control mice that received saline. ${ }^{*} \mathrm{p}<0.05$ : as-miR-937-MSCs vs MSCs. \#p $<0.05$. MSCs vs saline. $\mathrm{N}=10$.
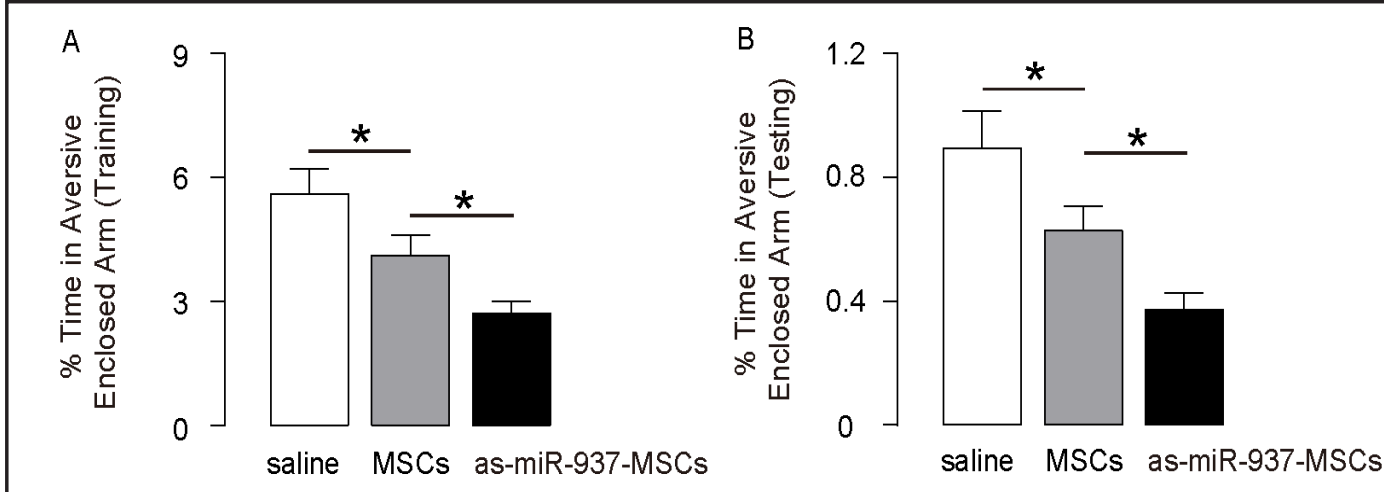

Fig. 4. Transplantation of as-miR-937-MSCs further improves the behavior of the AD mice in a Plus-Maze Discriminative avoidance Task (PM-DAT). The mice were then evaluated in a Plus-Maze Discriminative avoidance Task (PM-DAT). (A-B) Eepeated measurements of the percent time spent in the aversive enclosed arm parameter with treatment as a between-subject factor and time (minutes of observation) as a repeated measurement factor were performed in a training section (A), and in a testing section (B). ${ }^{*}<<0.05$. N=10.

behavior and innate interest (Fig. 3). The mice that received as-miR-937-MSCs mice showed further improvement in the test, compared to the mice that received MSCs, which already had better performance, compared to the control mice that received saline (Fig. 3). Together, these data suggest that transplantation of as-miR-937-MSCs further improves the recovery of Social Recognition Memory in AD mice.

Transplantation of as-miR-937-MSCs further improves the behavior of the AD mice in a Plus-Maze Discriminative avoidance Task (PM-DAT)

The mice were then evaluated in a Plus-Maze Discriminative avoidance Task (PMDAT). In the training session, repeated measurements of the percent time spent in the aversive enclosed arm parameter with treatment as a between-subject factor and time (minutes of observation) as a repeated measurement factor were performed. We found that transplantation of MSCs improved the acquisition deficits of the task, compared to controls, while the transplantation of as-miR-937-MSCs further improved it (Fig. 4A-B). Together, these data suggest that transplantation of as-miR-937-MSCs further improves the behavior of the AD mice in a PM-DAT. 
A

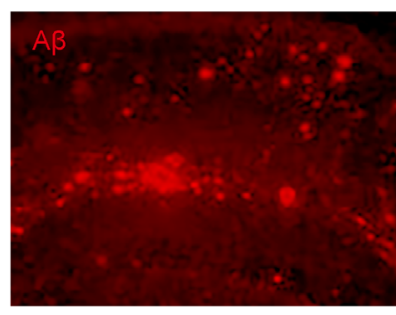

saline

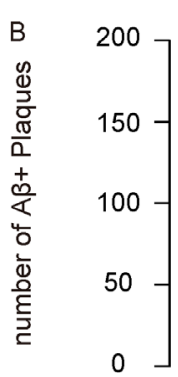

0

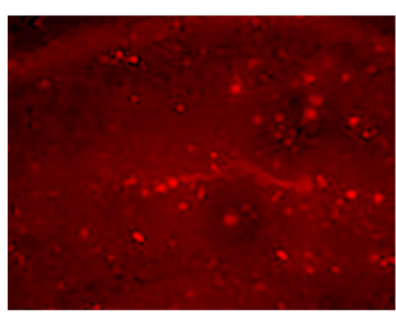

MSCs

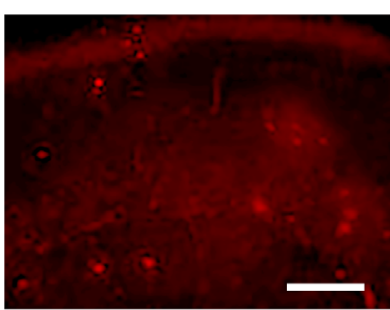

as-miR-937-MSCs
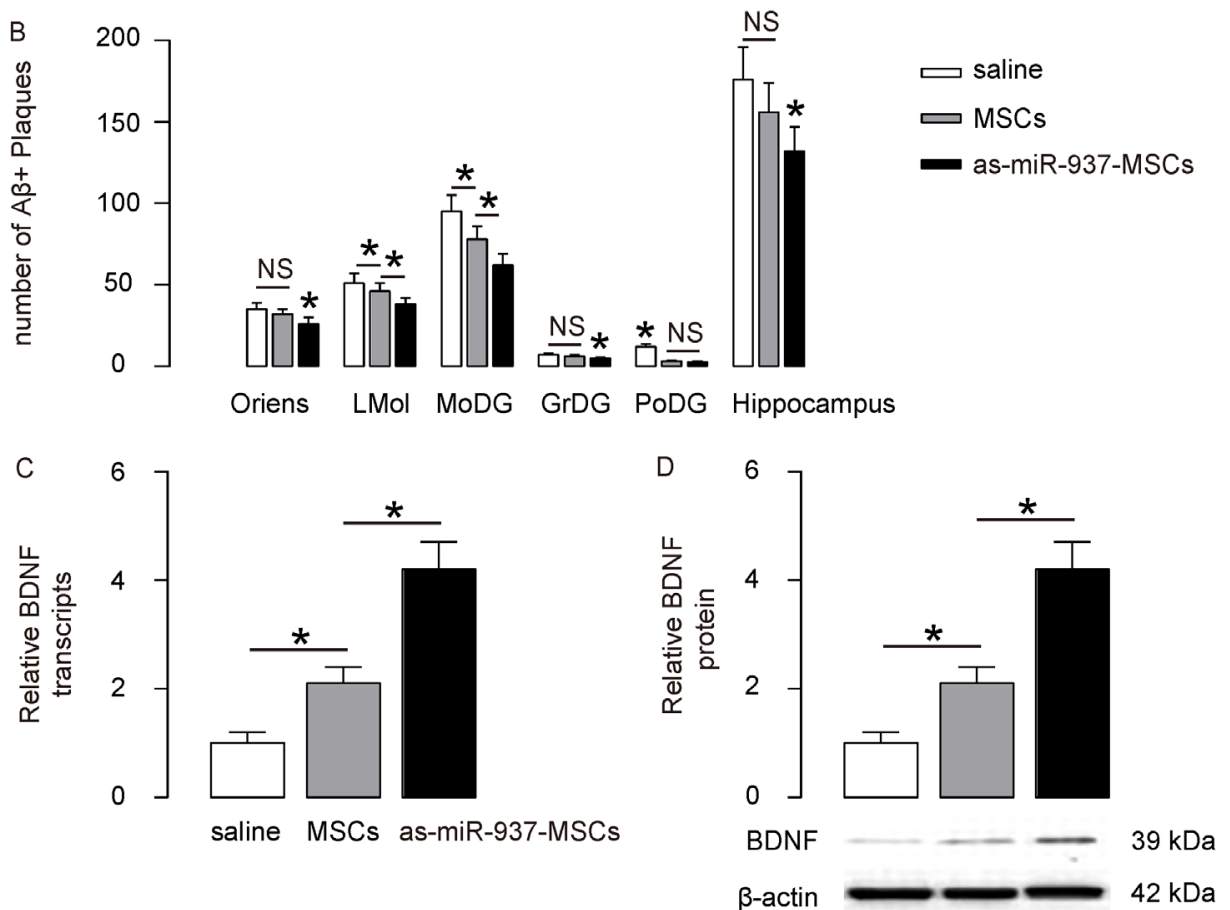

saline MSCs as-miR-937-MSCs

Fig. 5. Transplantation of as-miR-937-MSCs further reduces the number of A $\beta$ plaques and increases levels of BDNF in the brain of AD mice. (A) To identify and quantify senile plaques (A $\beta$ plaques) in the hippocampus, immunohistochemistry was performed using the Aß6E10 antibody, which reacted with the 1-16 residue amino acids of the human $A \beta$ protein. (B) To evaluate the effect of the treatments in diminishing the number and the distribution of the $A \beta$ plaques of the hippocampus of $\mathrm{AD}$ mice, we quantified the senile plaques in the hippocampal stratum oriens (Oriens), lacunosum molecular (LMol), molecular (MoDG), granular (GrDG) and polymorphic (PoDG) layers. The 3 experimental groups were then compared, showing that the number of $A \beta$ plaques significantly decreased by transplantation of as-miR-937-MSCs in Oriens and GrDG, while transplantation of MSCs had no significantly effects. Moreover, the number of A $\beta$ plaques significantly decreased by transplantation of MSCs, and further decreased in as-miR-937-MSCs-transplanted $\mathrm{AD}$ mice in LMol and MoDG. Further, the number of $\mathrm{A} \beta$ plaques significantly decreased by transplantation of either MSCs, or as-miR-937-MSCs in PoDG. Put together, the number of A $\beta$ plaques significantly decreased by transplantation of as-miR-937-MSCs in hippocampus, compared to transplantation of MSCs. (C) BDNF levels in mouse brain by mRNA (C), and by Western blot (D). ${ }^{*} p<0.05$. NS: non-significant. $N=10$. Scale bar is $100 \mu \mathrm{m}$.

Transplantation of as-miR-937-MSCs further reduces the number of A $\beta$ plaques and increases levels of BDNF in the brain of AD mice

To identify and quantify senile plaques (A $\beta$ plaques) in the hippocampus, immunohistochemistry was performed using the A $36 \mathrm{E} 10$ antibody, which reacted with the 1-16 residue amino acids of the human $A \beta$ protein (Fig. 5A). To evaluate the effect of 


\section{Cellular Physiology Cell Physiol Biochem 2015;37:321-330 \begin{tabular}{l|l|l} 
DOI: 10.1159/000430356 2012015 & O 2015 . Karger AG, Basel \\
\hline
\end{tabular} and Biochemistry Publisnea onine: August 24, 2015_ www.karger.com/cpb \\ Liu/Wang/Wang/Xu: Therapy of AD by As-MiR-937-Expressing MSCs}

the treatments in diminishing the number and the distribution of the $A \beta$ plaques of the hippocampus of $\mathrm{AD}$ mice, we quantified the senile plaques in the hippocampal stratum oriens (Oriens), lacunosum molecular (LMol), molecular (MoDG), granular (GrDG) and polymorphic (PoDG) layers. The 3 experimental groups were then compared, showing that the number of $A \beta$ plaques significantly decreased by transplantation of as-miR-937MSCs in Oriens and GrDG, while transplantation of MSCs had no significant effects (Fig. 5B). Moreover, the number of $A \beta$ plaques significantly decreased by transplantation of MSCs, and further decreased in as-miR-937-MSCs-transplanted AD mice in LMol and MoDG (Fig. 5B). Further, the number of $A \beta$ plaques significantly decreased by transplantation of either MSCs, or as-miR-937-MSCs in PoDG (Fig. 5B). Put together, the number of A $\beta$ plaques significantly decreased by transplantation of as-miR-937-MSCs in the hippocampus, compared to transplantation of MSCs (Fig. 5B).

We then examined the levels of a neuroregenerative factor, BDNF, in the mouse brain in these mice. BDNF is good marker for neuroregeneration. We found that in AD mice, the levels of BDNF significantly increased by transplantation of MSCs, and further increased by transplantation of as-miR-937-MSCs, by mRNA (Fig. 5C), and by Western blot (Fig. 5D).

Together, these data suggest that depletion of miR-937 in transplanted MSCs improves neuroregeneration and prevents neurodegradation.

\section{Discussion}

Our data suggest that the depletion of miR-937 in MSCs significantly improves the effects of MSC transplantation on neuroregeneration in AD mice, significantly recovers the innate interest in novelty and counteracts the social and discriminative type-memories and learning deficits present in $\mathrm{AD}$ mice. The social recognition paradigm is a model of social memory dependent on hippocampal function that can be used in pathophysiological processes, such as ischemia and aging, which are known to interfere with these processes.

In our study, using this resident-intruder paradigm, the transplantation of miR-937modified MSCs was able to improve the social recognition memory and recover the novelty component of short-term memory impaired in AD mice. In the PM-DAT, the avoidance of the aversive enclosed arm upon testing has been validated as a measurement of retention, since the amnestic manipulation decreases this effect. Using this animal model, we demonstrate that in the training session AD mice decrease learning levels, as demonstrated by the increased time spent in the aversive enclosed arm. MSCs attenuated and as-miR-937-MSCs abolished this learning deficit. In a testing session, AD mice showed impaired retrieval of the memory task. MSCs improved and as-miR-937-MSCs further improved the recover memory from deficits present in AD mice.

The depletion of miR-937 in MSCs increases the protein translation of Brn-4 in MSCs, which may be responsible for all the observed results. However, the effects of miR-937 on MSCs may not only be conducted through Brn-4, since its 3'-UTR of mRNA targets may include other factors than Brn-4. In the future, further analyses of these factors may provide a more complete understanding of the role of miR-937 in MSCs.

\section{Acknowledgements}

This work was supported by Medical Science and Technology Development Plan of Shandong Province (2014WS0239) and Youth Fund of the 2nd Hospital of Shandong University (Y2013010021).

\section{Disclosure Statement}

The authors have declared that no competing interests exist.

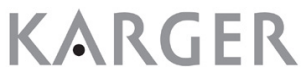




\section{Cellular Physiology Cell Physiol Biochem 2015;37:321-330 \begin{tabular}{ll|l} 
and Biochemistry & $\begin{array}{l}\text { DOI: 10.1159/000430356 } \\
\text { Publisned onlne: August 24, } 2015\end{array}$ & $\begin{array}{l}\text { O) 2015 S. Karger AG, Basel } \\
\text { www.karger.com/cpb }\end{array}$ \\
\cline { 2 - 3 }
\end{tabular}}

Liu/Wang/Wang/Xu: Therapy of AD by As-MiR-937-Expressing MSCs

\section{References}

1 Grimm MO, Hundsdorfer B, Grosgen S, Mett J, Zimmer VC, Stahlmann CP, Haupenthal VJ, Rothhaar TL, Lehmann J, Patzold A, Zinser EG, Tanila H, Shen J, Muller U, Grimm HS, Hartmann T: Ps dependent app cleavage regulates glucosylceramide synthase and is affected in alzheimer's disease. Cell Physiol Biochem 2014;34:92-110.

- Peng D, Pan X, Cui J, Ren Y, Zhang J: Hyperphosphorylation of tau protein in hippocampus of central insulinresistant rats is associated with cognitive impairment. Cell Physiol Biochem 2013;32:1417-1425.

3 Wang HM, Zhang T, Huang JK, Sun XJ: 3-n-butylphthalide (nbp) attenuates the amyloid-beta-induced inflammatory responses in cultured astrocytes via the nuclear factor-kappab signaling pathway. Cell Physiol Biochem 2013;32:235-242.

- 4 He J, Qiao JP, Zhu S, Xue M, Chen W, Wang X, Tempier A, Huang Q, Kong J, Li XM: Serum beta-amyloid peptide levels spike in the early stage of alzheimer-like plaque pathology in an app/ps1 double transgenic mouse model. Curr Alzheimer Res 2013;10:979-986.

-5 Fabelo N, Martin V, Marin R, Santpere G, Aso E, Ferrer I, Diaz M: Evidence for premature lipid raft aging in app/ps1 double-transgenic mice, a model of familial alzheimer disease. J Neuropathol Exp Neurol 2012;71:868-881.

6 Danielyan L, Klein R, Hanson LR, Buadze M, Schwab M, Gleiter CH, Frey WH: Protective effects of intranasal losartan in the app/ps1 transgenic mouse model of alzheimer disease. Rejuvenation Res 2010;13:195-201.

7 Trinchese F, Liu S, Battaglia F, Walter S, Mathews PM, Arancio O: Progressive age-related development of alzheimer-like pathology in app/ps1 mice. Ann Neurol 2004;55:801-814.

8 Czaja K, Fornaro M, Geuna S: Neurogenesis in the adult peripheral nervous system. Neural Regen Res 2012;7:1047-1054.

- Kim HJ, Sun W: Adult neurogenesis in the central and peripheral nervous systems. Int Neurourol J 2012;16:57-61.

10 Miles DK, Kernie SG: Hypoxic-ischemic brain injury activates early hippocampal stem/progenitor cells to replace vulnerable neuroblasts. Hippocampus 2008;18:793-806.

11 Brinton RD, Wang JM: Therapeutic potential of neurogenesis for prevention and recovery from alzheimer's disease: Allopregnanolone as a proof of concept neurogenic agent. Curr Alzheimer Res 2006;3:185-190.

12 Zhao C, Deng W, Gage FH: Mechanisms and functional implications of adult neurogenesis. Cell 2008;132:645-660.

13 Jiang J, Bu X, Liu M, Cheng P: Transplantation of autologous bone marrow-derived mesenchymal stem cells for traumatic brain injury. Neural Regen Res 2012;7:46-53.

14 Munoz JR, Stoutenger BR, Robinson AP, Spees JL, Prockop DJ: Human stem/progenitor cells from bone marrow promote neurogenesis of endogenous neural stem cells in the hippocampus of mice. Proc Natl Acad Sci U S A 2005;102:18171-18176.

15 Kan I, Barhum Y, Melamed E, Offen D: Mesenchymal stem cells stimulate endogenous neurogenesis in the subventricular zone of adult mice. Stem Cell Rev 2011;7:404-412.

16 Shin KK, Kim YS, Kim JY, Bae YC, Jung JS: Mir-137 controls proliferation and differentiation of human adipose tissue stromal cells. Cell Physiol Biochem 2014;33:758-768.

$>17$ Chou WW, Wang YT, Liao YC, Chuang SC, Wang SN, Juo SH: Decreased microrna-221 is associated with high levels of tnf-alpha in human adipose tissue-derived mesenchymal stem cells from obese woman. Cell Physiol Biochem 2013;32:127-137.

18 Zhang X, Zhang L, Cheng X, Guo Y, Sun X, Chen G, Li H, Li P, Lu X, Tian M, Qin J, Zhou H, Jin G: Igf-1 promotes brn-4 expression and neuronal differentiation of neural stem cells via the pi3k/akt pathway. PLoS One 2014;9:e113801.

19 Tan XF, Qin JB, Jin GH, Tian ML, Li HM, Zhu HX, Zhang XH, Shi JH, Huang Z: Effects of brn-4 on the neuronal differentiation of neural stem cells derived from rat midbrain. Cell Biol Int 2010;34:877-882.

20 Shi J, Jin G, Zhu H, Tian M, Zhang X, Qin J, Tan X: The role of brn-4 in the regulation of neural stem cell differentiation into neurons. Neurosci Res 2010;67:8-17.

-21 Zhang X, Jin G, Wang L, Hu W, Tian M, Qin J, Huang H: Brn-4 is upregulated in the deafferented hippocampus and promotes neuronal differentiation of neural progenitors in vitro. Hippocampus 2009;19:176-186. 